\title{
TOX3 is a favorable prognostic indicator and potential immunomodulatory factor in lung adenocarcinoma
}

\author{
DE ZENG ${ }^{1}$, HAOYU LIN ${ }^{2}$, JIANXIONG CUI ${ }^{2}$ and WEIQUAN LIANG ${ }^{2}$ \\ ${ }^{1}$ Department of Medical Oncology, Cancer Hospital of Shantou University Medical College; ${ }^{2}$ Department of Breast and \\ Thyroid Surgery, The First Affiliated Hospital of Shantou University Medical College, Shantou, Guangdong 515000, P.R. China
}

Received February 13, 2018; Accepted January 14, 2019

DOI: $10.3892 / \mathrm{ol} .2019 .10748$

\begin{abstract}
Thymocyte selection-associated high mobility group box (TOX) genes represent a novel family of genes. Deregulated expression of TOXs has been reported in a variety of cancer types, including lung cancer. It has also been reported that TOXs are crucial regulators of the immune system. The present study systematically evaluated the prognostic values of TOX family members using a set of publicly accessible databases, including Oncomine, Kaplan-Meier plotter and cBioPortal. It was revealed that TOX expression profiles differed between lung cancer and normal tissues, and high expression of TOX mRNAs generally predicted improved survival outcomes. Notably, TOX3 expression was significantly increased in lung adenocarcinoma, compared with other pathological subtypes of lung cancer. Survival analysis demonstrated that elevated TOX3 expression was significantly associated with improved progression-free and overall survival in patients with lung adenocarcinoma. Furthermore, correlation analysis indicated that TOX3 expression was negatively correlated with the expression of programmed death-1 receptor (PD-1), PD-ligand 1 and Hepatitis A virus cellular receptor 2 in lung adenocarcinoma. These results indicated that TOX 3 is a prognostic indicator and promising immunomodulatory factor in lung adenocarcinoma. Future studies investigating the role of TOX3 in lung cancer immunity are warranted.
\end{abstract}

\section{Introduction}

The development of targeted therapy has resulted in improved lung cancer treatment over the past decade $(1,2)$. Recently, with the identification of immunity-associated targets such as programmed death-1 receptor (PD-1), PD-ligand 1 (PD-L1)

Correspondence to: Dr De Zeng, Department of Medical Oncology, Cancer Hospital of Shantou University Medical College, 7 Raoping Road, Shantou, Guangdong 515000, P.R. China E-mail: alexzengde@163.com

Key words: thymocyte selection-associated high mobility group box, lung adenocarcinoma, prognostic value, immunomodulatory factor and cytotoxic T lymphocyte-associated protein 4 (CTLA4), as well as the development of novel immunotherapy agents, lung cancer treatments have improved notably in terms of disease control and survival outcomes (3). However, the success rate of immunotherapy remains low, and a large number of cancer types, including lung cancer, remain largely insensitive or refractory to PD-1, PD-L1 and CTLA4 blockade $(2,4-6)$. Therefore, research has focused on identifying novel immunotherapy targets or immunomodulatory factors that can be exploited to enhance the response to current immunotherapeutic agents and address immunotherapy resistance in cancer $(7,8)$.

Thymocyte selection-associated high mobility group box factors (TOXs) are members of an evolutionarily conserved DNA-binding protein family, and are expressed in several immune-relevant cell subsets (9). Increasing evidence has demonstrated that TOX gene family members are aberrantly expressed or mutated in various human diseases, particularly in malignancies, such as breast cancer (10). For example, studies showed that TOX3 had a strong correlation with the development of breast cancer (11). TOXs have been reported to serve a pivotal role in malignant cluster of differentiation 4-positive $\mathrm{T}$ cell development and are overexpressed in cytotoxic $\mathrm{T}$ cell lymphoma (12). A previous study by Tessema et al (13) demonstrated that TOX1 is hypermethylated in breast cancer tissues but not in adjacent normal tissues, indicating that TOX1 may be a novel tumor biomarker. Additionally, it was reported that TOX2 is unmethylated in normal cells, while it was methylated in lung and breast tumors (13). Zhang et al (14) reported that TOX3 rs3803662 is associated with a significantly favorable prognosis in patients with diffuse-type gastric cancer. Using co-expression analysis of long non-coding RNAs and mRNAs in breast cancer, Wu et al (15) proposed that TOX4 is correlated with breast tumorigenesis and clinical outcomes. Nevertheless, the prognostic value and potential roles of individual TOX family members in lung cancer remain to be fully characterized.

In the present study, large databases were evaluated in order to determine the prognostic value of different TOX family members in lung cancer. The results indicated that characterizing the TOX expression status in patients with lung cancer may be valuable for diagnostic and prognostic assessment, as well as guiding the management of lung cancer treatment in the future. 


\section{Materials and methods}

Oncomine analysis. The expression of TOX family members mRNA in different cancer types was determined by analyzing the Oncomine database (www.oncomine.org), which is an online, publicly accessible cancer microarray database aimed to facilitate the identification of potential target genes based on genome-wide expression analyses $(16,17)$.

In the present study, Student's t-test was used to generate a P-value for comparisons between cancer specimens and normal control datasets. The fold-change was defined as $\geq 2$ and P-value of $<0.01$ was used as the cut-off point, as described in our previous study (18).

Kaplan-Meier plotter survival analysis. The prognostic values of those TOX family members that were observed to be highly expressed in lung samples were further assessed by displaying the corresponding first progression (FP) and overall survival (OS) curves using Kaplan-Meier plotter (www.kmplot.com). The log-rank P-value was calculated and depicted on the webpage (19).

Correlation analysis using cBioPortal for cancer genomics. Correlations between the expression of TOX family members and that of PD-1, PD-L1 and Hepatitis A virus cellular receptor 2 (HAVCR2) in lung cancer were analyzed using the cBioPortal database (http://www.cbioportal.org/index.do) (20). Lung adenocarcinoma [The Cancer Genome Atlas (TCGA), Provisional] and lung squamous cell carcinoma (TCGA, Provisional) datasets were used (cbioportal.org/datasets). Data are presented as the mean \pm standard error of the mean. Correlation coefficients between mRNA levels were obtained through Pearson's correlation analysis. Statistical analysis was performed using SPSS 23.0 (IBM Corp., Armonk, NY, USA), and $\mathrm{P}<0.05$ was considered to indicate a statistically significant difference.

\section{Results}

mRNA expression patterns of TOX family members in human lung cancer. To date, four TOX family members (TOX1-4) have been identified in human cancer types (10). In the present study, Oncomine analysis was used to investigate the differences in mRNA expression profiles of these four TOXs between tumor and normal tissues in various types of cancer. As depicted in Fig. 1, the database contained a total of 429, 273, 382 and 416 unique analyses for TOX1, TOX2, TOX3 and TOX4, respectively. A total of three studies demonstrating a significant increase in mRNA expression levels for TOX1 in lung cancer, compared with normal tissues, were found. For TOX2, all five datasets revealed significantly increased levels of TOX2 in normal tissues, compared with the lung cancer tissues. A total of nine analyses reported increased expression of TOX3 in tumors, while only one study reported a significant decrease in TOX3 expression in tumors, compared with normal tissues. These data indicate that TOX1 and TOX3 mRNA expression is significantly increased in lung cancer, compared with normal tissues (Fig. 1).

TOX3 is exclusively overexpressed in lung adenocarcinoma. Oncomine analysis revealed that, among all the TOXs,
TOX3 was significantly upregulated in lung adenocarcinoma, compared with normal samples across a wide variety of datasets. The dataset from the study by Stearman et al (21) revealed that TOX3 is elevated 12.617-fold in lung adenocarcinoma samples, compared with normal samples $\left(\mathrm{P}=2.17 \times 10^{-7}\right.$; Fig. 2A). Furthermore, Landi et al (22) observed that TOX3 is overexpressed 4.968 -fold in adenocarcinoma samples, compared with normal lung tissues $\left(\mathrm{P}=2.800 \times 10^{-18}\right.$; Fig. $\left.2 \mathrm{~B}\right)$. In another dataset with 246 samples from the study by Garber et al (23), TOX3 expression was increased 8.685-fold in adenocarcinoma, compared with normal lung tissue $\left(\mathrm{P}=1.180 \times 10^{-22}\right.$; Fig. 2C).

The expression profiles of TOX1 were varied in other pathological subtypes of lung cancer. In small cell lung cancer and lung carcinoid tumor, TOX1 expression was 5.591 and 11.468 times increased, compared with normal lung tissue, respectively (Fig. 2D and E) (19). Another dataset from the study by Hou et al (24) demonstrated that TOX1 expression was 3.416 times increased in large cell lung carcinoma, compared with lung adenocarcinoma (Fig. 2F).

Elevated TOXs expression predicts longer survival time in patients with lung cancer. The prognostic value of TOXs in lung cancer was analyzed using the Kaplan-Meier plotter database. FP and OS were analyzed for each TOX gene. In patients with lung cancer, increased FP was associated with increased TOX1 [Fig. 3A; hazard ratio $(\mathrm{HR})=0.800 ; \mathrm{P}=0.020]$, TOX2 (Fig. 3B; HR=0.700; $\mathrm{P}=0.011$ ), TOX3 (Fig. 3C; HR=0.780; $\mathrm{P}=9.500 \times 10^{-3}$ ) and TOX4 (Fig. 3D; HR=0.720; $\mathrm{P}=7.500 \times 10^{-4}$ ) expression. These results demonstrated that high expression of all TOXs was associated with improved survival outcomes in patients with lung cancer.

Consistently, improved OS in patients with lung cancer was also associated with increased TOX1 (Fig. 3E; HR=0.750; $\mathrm{P}=1.500 \times 10^{-5}$ ), TOX2 (Fig. 3F; HR=0.600; $\mathrm{P}=9.500 \times 10^{-10}$ ), TOX3 (Fig. 3G; HR=0.680; $\mathrm{P}=1.300 \times 10^{-9}$ ) and TOX4 (Fig. 3H; $\left.\mathrm{HR}=0.670 ; \mathrm{P}=3.400 \times 10^{-10}\right)$ mRNA expression. These data revealed that elevated TOXs expression predicted improved prognosis in patients with lung cancer, indicating that TOXs potentially act as tumor suppressors in lung cancer.

Elevated TOX3 expression is associated with distinctive survival outcomes in lung adenocarcinoma. Subsequently, the prognostic significance of each TOX was stratified by a number of default clinicopathological parameters, including smoking status and chemotherapy status, in the Kaplan-Meier plotter database was examined. The prognostic effects of the four genes are detailed in Tables I and II. The results revealed that high TOX3 mRNA expression was significantly associated with improved FP in a subgroup of patients who had never smoked $\left(\mathrm{HR}=0.370 ; \mathrm{P}=7.500 \times 10^{-5}\right.$; Table I). By contrast, high mRNA TOX4 expression was significantly associated with improved FP both in patients with smoking $(\mathrm{HR}=0.730 ; \mathrm{P}=0.012)$ and without smoking history ( $\mathrm{HR}=0.560 ; \mathrm{P}=0.018$ ) (Table I). Notably, the present data also demonstrated that high levels of TOX3 mRNA were significantly associated with increased OS in patients who had received chemotherapy $\left(\mathrm{HR}=0.580 ; \mathrm{P}=8.200 \times 10^{-3}\right.$; Table II), indicating a potential role of TOX3 in the chemosensitivity of lung cancer. 


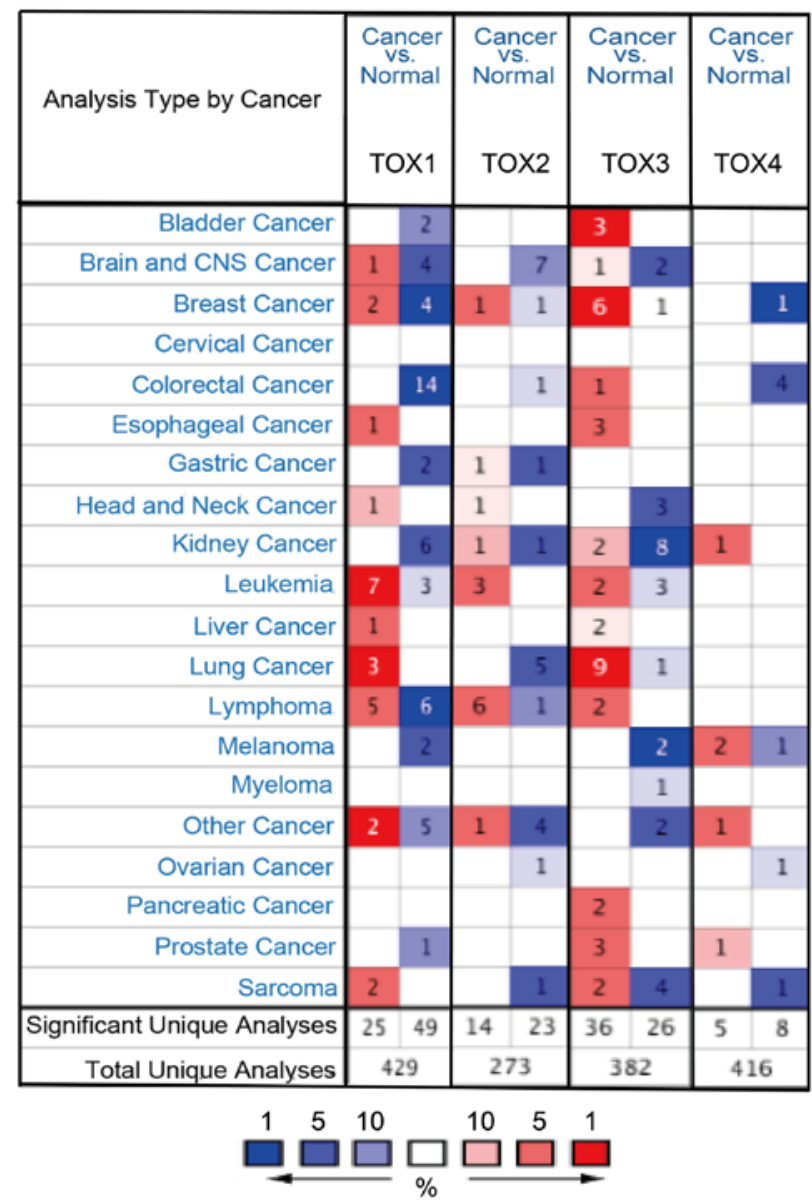

Figure 1. The mRNA expression pattern of TOX family members in different tumor types. This figure depicts the number of datasets with statistically significant mRNA overexpression (red) or downregulated expression (blue) of the target gene (cancer vs. normal). The P-value threshold is 0.01 . The number in each cell represents the number of analyses that meet the threshold within those analysis and cancer types. The gene rank was analyzed by percentile of target gene in the top of all genes measured in each research. Cell color is determined by the best gene rank percentile for the analyses within the cell. CNS, central nervous system; TOX, thymocyte selection-associated high mobility group box.

A

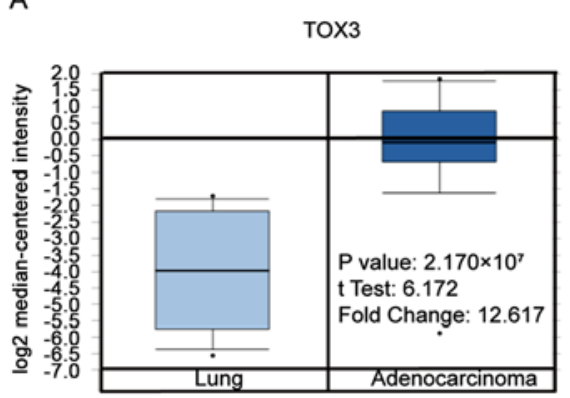

D

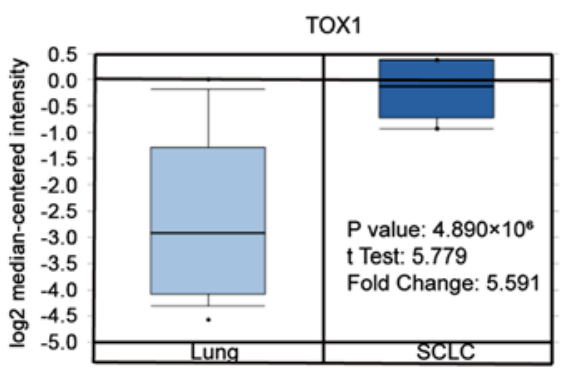

B

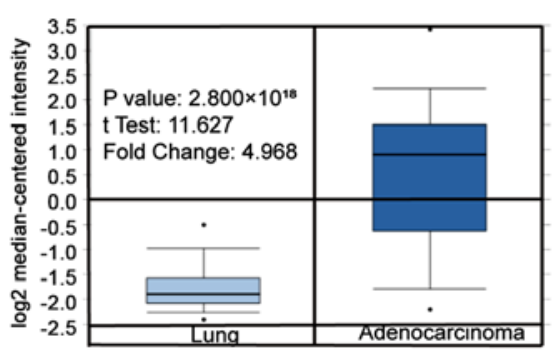

$\mathrm{E}$

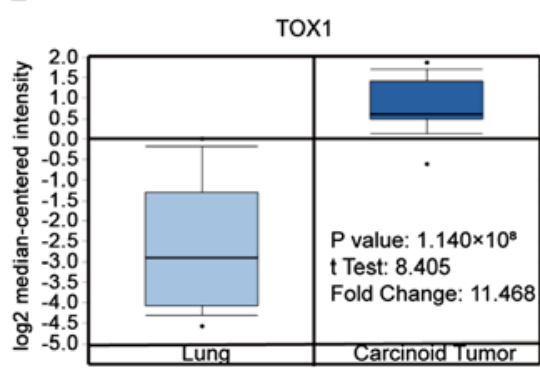

C

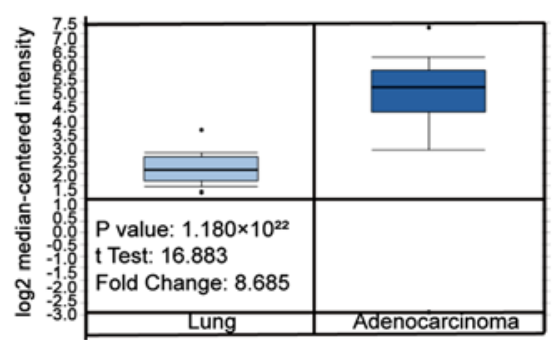

$\mathrm{F}$

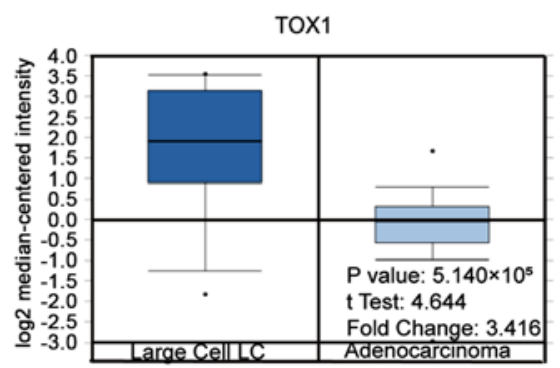

Figure 2. TOX3 is overexpressed in lung adenocarcinoma. Box plots derived from gene expression data in Oncomine comparing expression of a specific TOX family member in lung adenocarcinoma and normal lung tissues. Comparison of the mRNA expression levels of TOX3 with (A) Stearman et al (21) (B) Landi et al (22) and (C) Garber et al (23). Comparison of TOX1 mRNA expression between (D) small cell lung cancer and normal lung tissue; (E) carcinoid tumor and normal lung tissue; and $(\mathrm{F})$ between large cell lung cancer and lung adenocarcinoma. 
A

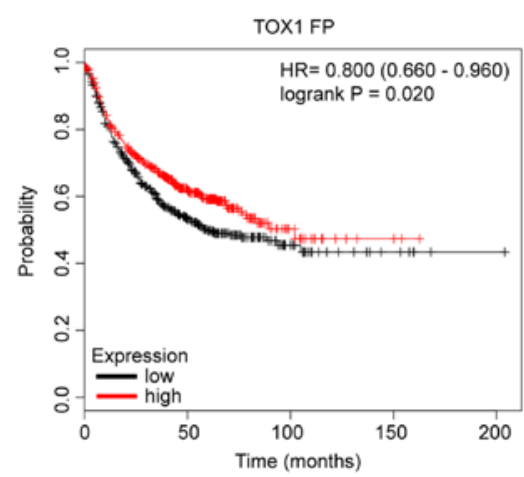

C

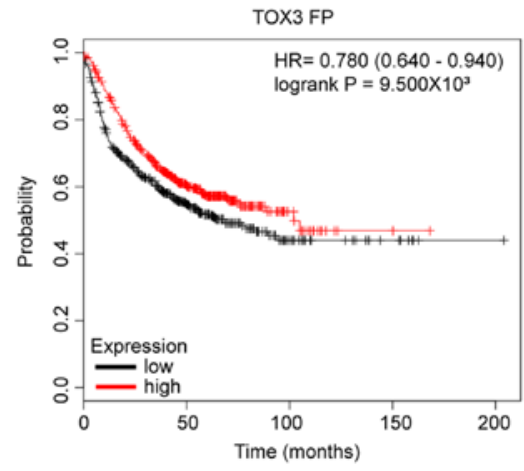

$E$

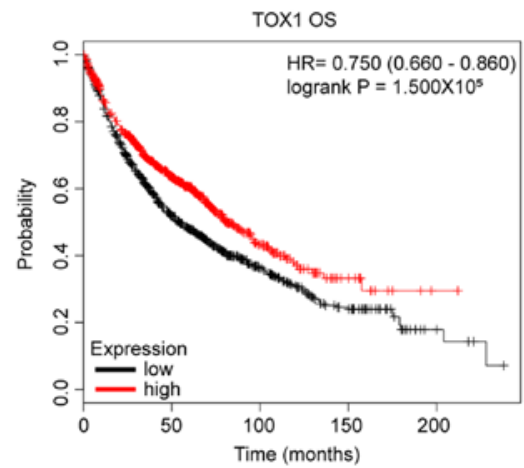

G

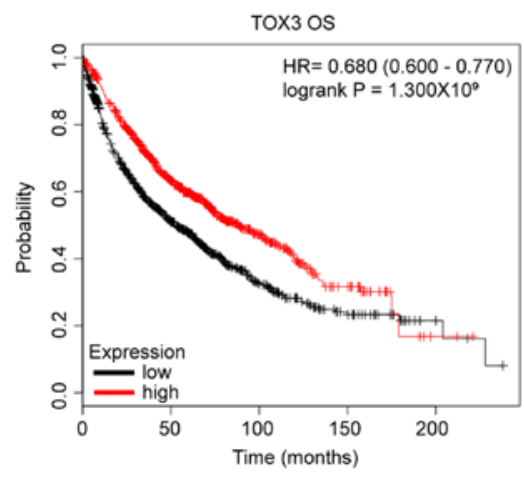

B

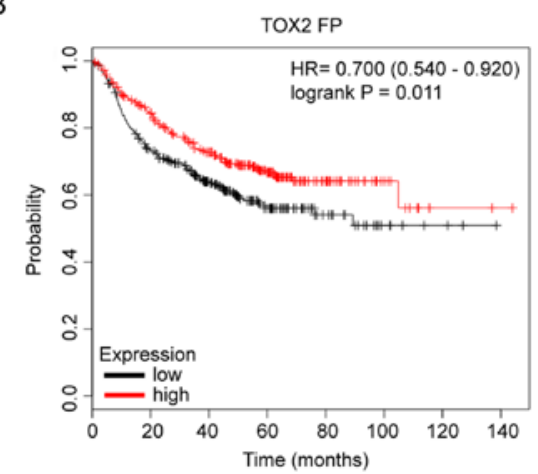

D

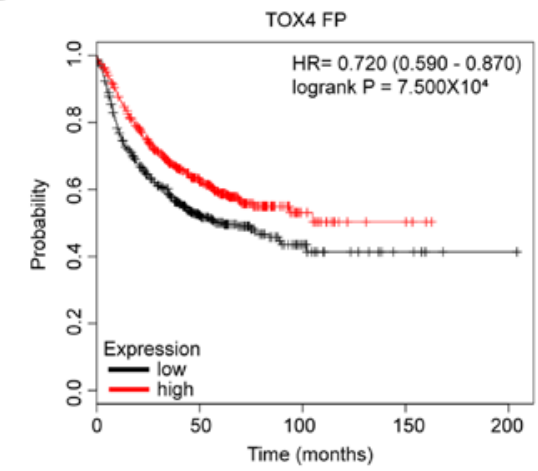

$\mathrm{F}$

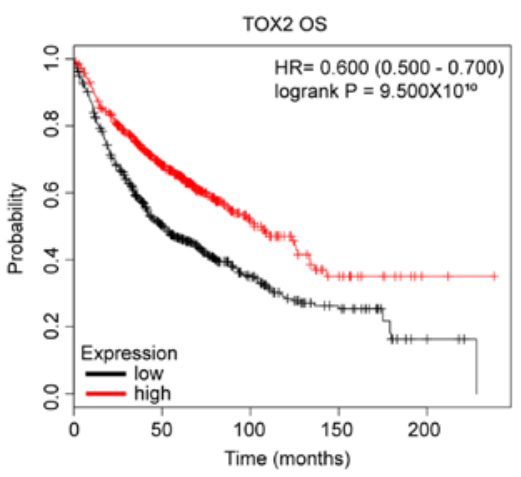

$\mathrm{H}$

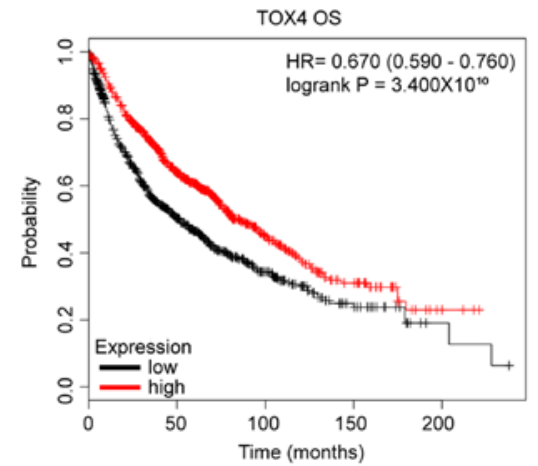

Figure 3. Elevated TOX expression predicts improved survival in patients with lung cancer. Kaplan-Meier analysis of FP in all patients with lung cancer based on (A) TOX1, (B) TOX2, (C) TOX3, and (D) TOX4 mRNA expression levels. Kaplan-Meier analysis of OS in all patients with lung cancer based on (E) TOX1, (F) TOX2, (G) TOX3, and (H) TOX4 mRNA expression levels. TOX, thymocyte selection-associated high mobility group box; OS, overall survival; FP, first progression; HR, hazard ratio.

Notably, the present results revealed that high TOX3 mRNA expression is significantly associated with improved FP $\left(\mathrm{HR}=0.600 ; \mathrm{P}=1.300 \times 10^{-3}\right.$; Fig. $\left.4 \mathrm{~A}\right)$ and $\mathrm{OS}(\mathrm{HR}=0.590$; $\mathrm{P}=9.700 \times 10^{-6}$; Fig. $4 \mathrm{~B}$ ) in patients with lung adenocarcinoma.
However, no significant difference was observed in $\mathrm{FP}(\mathrm{HR}=1.180$; $\mathrm{P}=0.520$; Fig. $4 \mathrm{C})$ or $\mathrm{OS}(\mathrm{HR}=0.920 ; \mathrm{P}=0.460$; Fig. 4D) in patients with squamous cell carcinoma. Subgroup analysis, according to smoking status, demonstrated that TOX3 expression 
Table I. Association of FP in TOX family mRNA expression with smoking status of patients with lung cancer.

\begin{tabular}{|c|c|c|c|c|c|}
\hline TOX family & Smoking status & Cases & HR & $95 \% \mathrm{CI}$ & P-value \\
\hline \multirow[t]{2}{*}{ TOX1 } & Smoke & 605 & 0.870 & $(0.680-1.110)$ & 0.270 \\
\hline & Never smoke & 193 & 0.640 & $(0.400-1.050)$ & 0.075 \\
\hline \multirow[t]{2}{*}{ TOX2 } & Smoke & 297 & 0.720 & $(0.490-1.070)$ & 0.110 \\
\hline & Never smoke & 141 & 0.600 & $(0.320-1.110)$ & 0.098 \\
\hline \multirow[t]{2}{*}{ TOX3 } & Smoke & 603 & 0.850 & $(0.670-1.090)$ & 0.200 \\
\hline & Never smoke & 193 & 0.370 & $(0.220-0.620)$ & $7.5 \times 10^{-5 a}$ \\
\hline \multirow[t]{2}{*}{ TOX4 } & Smoke & 603 & 0.730 & $(0.570-0.940)$ & $0.012^{\mathrm{a}}$ \\
\hline & Never smoke & 193 & 0.560 & $(0.340-0.910)$ & $0.018^{\mathrm{a}}$ \\
\hline
\end{tabular}

${ }^{\mathrm{a}} \mathrm{P}<0.05$. TOX, thymocyte selection-associated high mobility group box; HR, hazard ratio; CI, confidence interval; FP, first progression.

Table II. Association of OS in TOX family mRNA expression with chemotherapy status of patients with lung cancer.

\begin{tabular}{lccccc}
\hline TOX family & Chemotherapy status & Cases & HR & 95\% CI & P-value \\
\hline TOX1 & Yes & 176 & 0.890 & $0.590-1.340$ & 0.490 \\
& No & 310 & 0.810 & $0.580-1.140$ & 0.740 \\
TOX2 & Yes & 34 & 2.520 & $0.760-8.410$ & 0.220 \\
& No & 21 & 0.590 & $0.120-2.930$ & 0.520 \\
TOX3 & Yes & 310 & 0.960 & $0.690-1.340$ & 0.420 \\
& No & 176 & 0.580 & $0.380-0.870$ & $8.2 \times 10^{-33}$ \\
TOX4 & Yes & 125 & 0.780 & $0.510-1.180$ & 0.240 \\
& No & 310 & 0.750 & $0.530-1.040$ & 0.190 \\
\hline
\end{tabular}

${ }^{\text {a }}<0.05$. TOX, thymocyte selection-associated high mobility group box; HR, hazard ratio; CI, confidence interval; OS, overall survival.

was associated with significantly improved FP $(\mathrm{HR}=0.850$; $\mathrm{P}=0.020$; Fig. 4E) and $\mathrm{OS}\left(\mathrm{HR}=0.710 ; \mathrm{P}=1.200 \times 10^{-3}\right.$; Fig. 4F in smoking patients. TOX3 expression was also associated with increased FP $\left(\mathrm{HR}=0.370 ; \mathrm{P}=7.500 \times 10^{-5}\right.$; Fig. $\left.4 \mathrm{G}\right)$ and $\mathrm{OS}$ $\left(\mathrm{HR}=0.270 ; \mathrm{P}=1.700 \times 10^{-5}\right.$; Fig. $\left.4 \mathrm{H}\right)$ in non-smoking patients with lung cancer.

High mRNA expression of TOX3 is correlated with low $m R N A$ expression of PD-1, PD-L1 and HAVCR2 in lung adenocarcinoma. To further investigate the role of TOX3 in lung adenocarcinoma, the cBioPortal database was used to analyze the correlation between TOX3 and various immunotherapy target genes. Gene correlation analysis demonstrated that, in lung adenocarcinoma, high expression of TOX3 was correlated with low expression of PD-1 (Fig. 5A; r=-0.142; P=0.001), PD-L1 (Fig. 5C; r=-0.228; P<0.001) and HAVCR2 (Fig. 5E; $\mathrm{r}=-0.198 ; \mathrm{P}<0.001)$. However, in squamous cell carcinoma, no significant correlation was observed between TOX3 and PD-1 (Fig. 5B; r=-0.017; $\mathrm{P}=0.696$ ), PD-L1 (Fig. 5D; $\mathrm{r}=0.004$; $\mathrm{P}=0.930$ ) or HAVCR2 (Fig. 5F; $\mathrm{r}=-0.039 ; \mathrm{P}=0.384$ ) expression.

\section{Discussion}

Immunotherapy is increasingly being recognized as a key treatment for patients with lung cancer (8). The identification of novel immunomodulatory targets or factors is expected to aid a subset of patients with lung cancer, particularly those who do not benefit from the current therapy targeting immune checkpoint molecules, including PD-1, PD-L1 and CTLA-4 $(25,26)$.

TOX is novel gene family that serves a pivotal function in human immunity (9). Recently, deregulated expression of TOX family members has been reported in a wide range of human cancer types (10). For example, Seksenyan et al (27) reported two different roles for TOX3, one in the initiation of breast cancer, potentially associated with expression of TOX3 in mammary epithelial cell progenitors, and another role in the progression of cancer. Additionally, emerging studies indicated that, by monitoring TOX status in an individual tumor, the risk of cancer development and progression could be predicted, as could prognosis $(14,28)$. However, the potential roles and prognostic values of different TOX members remain to be fully clarified.

Through systematic and in-depth investigation of a series of databases, the present study identified the expression patterns of various TOX family members in lung cancer, and provided evidence to support their potential functions in lung cancer. Analysis of the Oncomine database indicated that, among all TOX members, TOX1 and TOX3 were significantly highly expressed in lung cancer, compared with normal controls, indicating important roles for these molecules in lung cancer. In a genome-wide comparison of DNA methylation between normal and tumor cells 
A

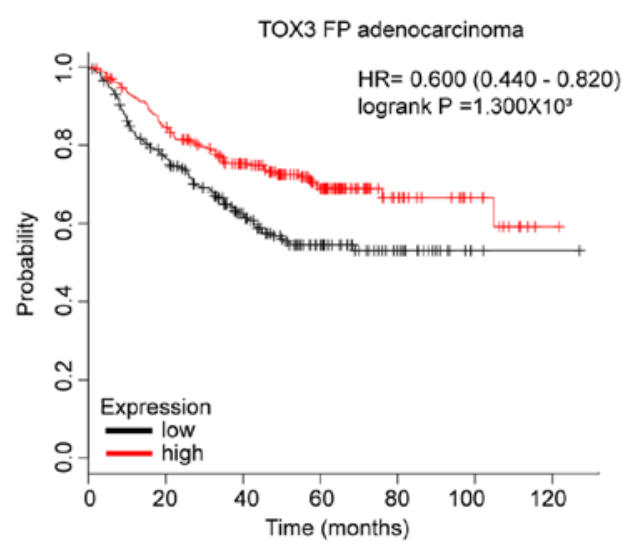

C

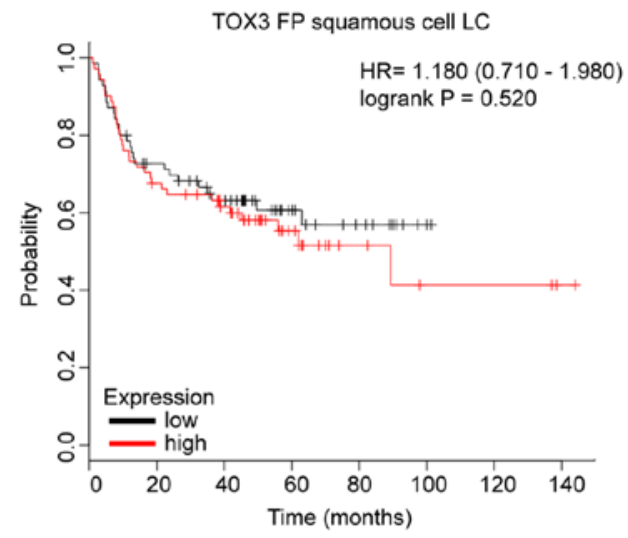

$\mathrm{E}$

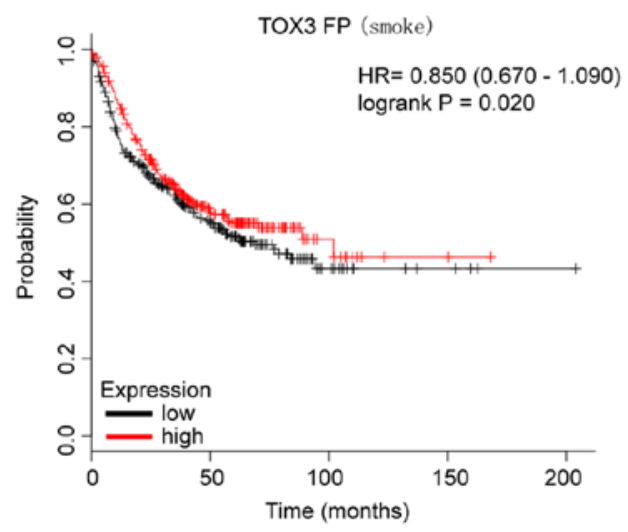

G

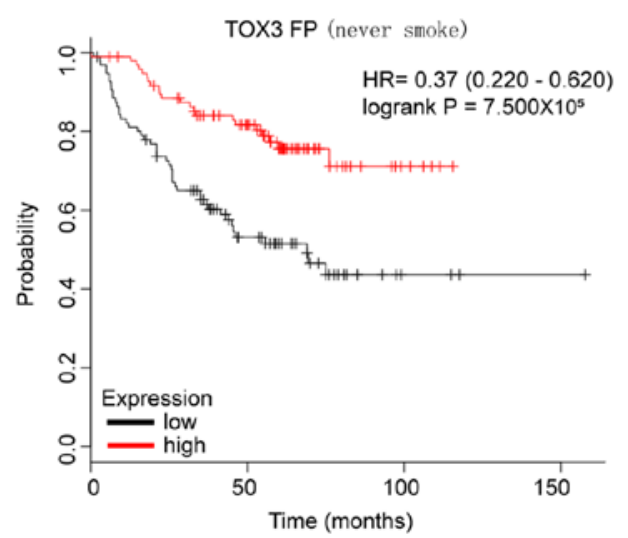

B

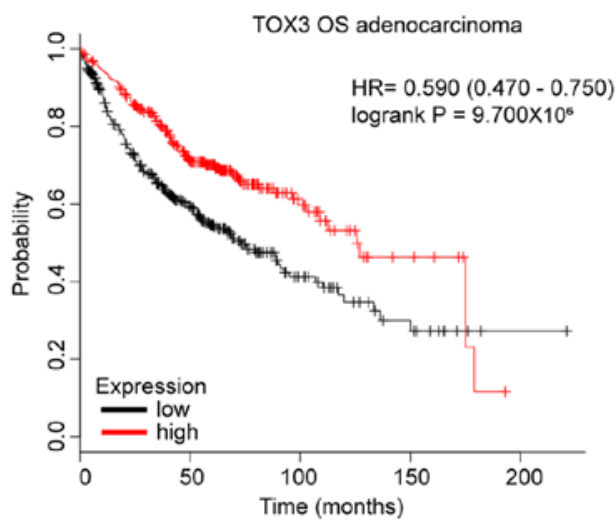

D

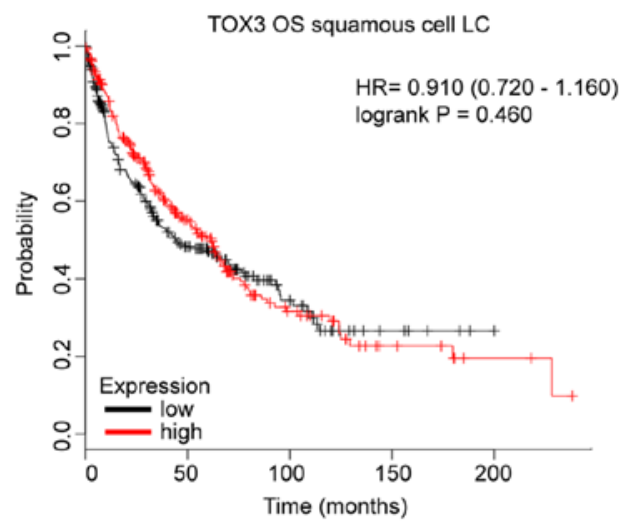

$\mathrm{F}$

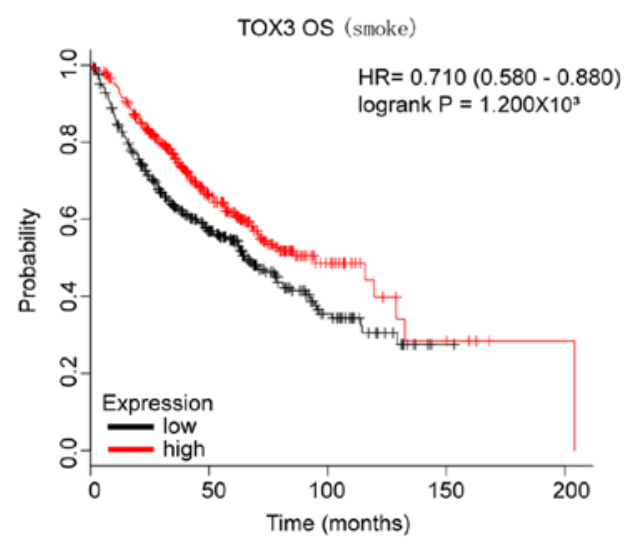

$\mathrm{H}$

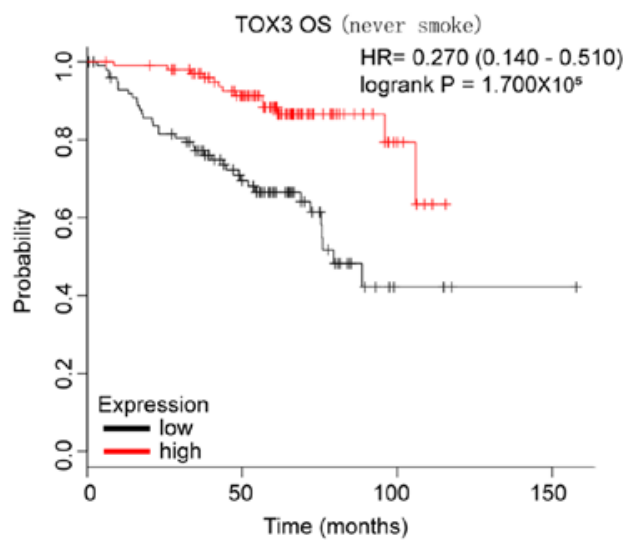

Figure 4. The prognostic values of TOX3 in lung adenocarcinoma. The association of TOX3 mRNA level and FP in patients with (A) lung adenocarcinoma or (B) squamous cell lung carcinoma. The association of TOX3 mRNA level and OS in patients with (C) lung adenocarcinoma or (D) squamous cell lung carcinoma. (E) Elevated TOX3 expression was associated with improved FP in smoking patients with lung adenocarcinoma. (F) Elevated TOX3 expression was associated with improved FP in non-smoking patients with lung adenocarcinoma. (G) Elevated TOX3 expression was associated with improved OS in smoking patients with lung adenocarcinoma. (H) Elevated TOX3 expression was associated with improved OS in non-smoking patients with lung adenocarcinoma. TOX, thymocyte selection-associated high mobility group box; OS, overall survival; FP, first progression; HR, hazard ratio; LC, lung carcinoma. 
A

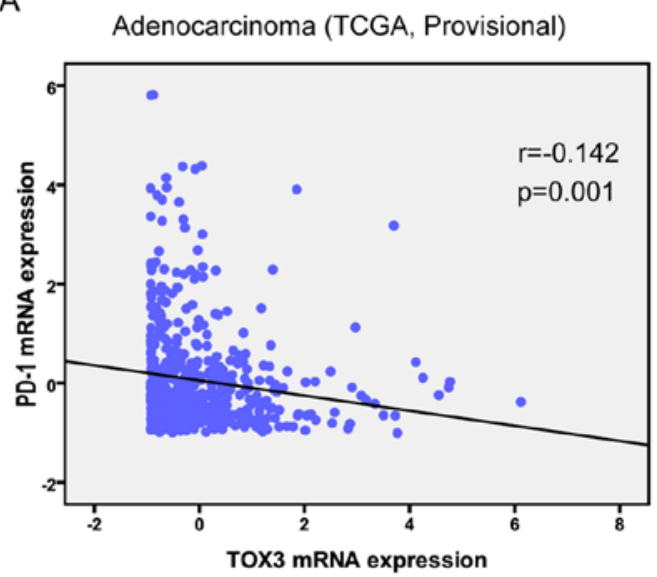

C

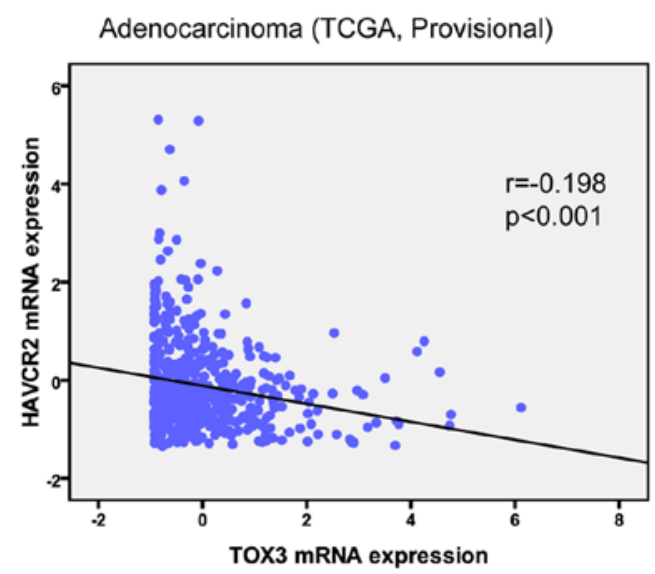

E

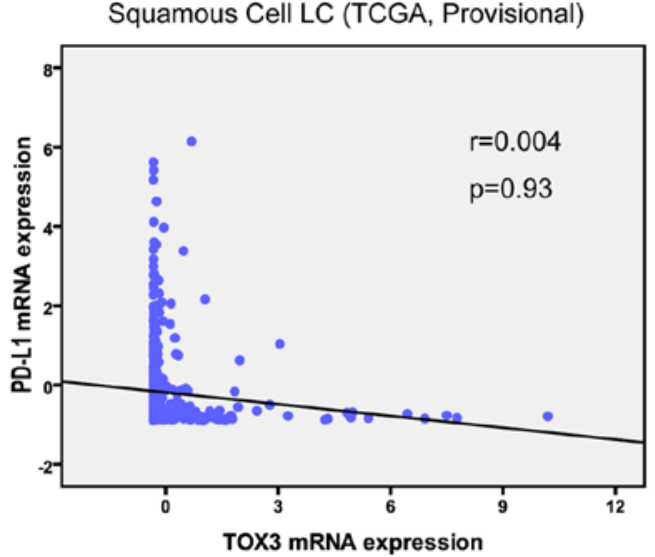

B

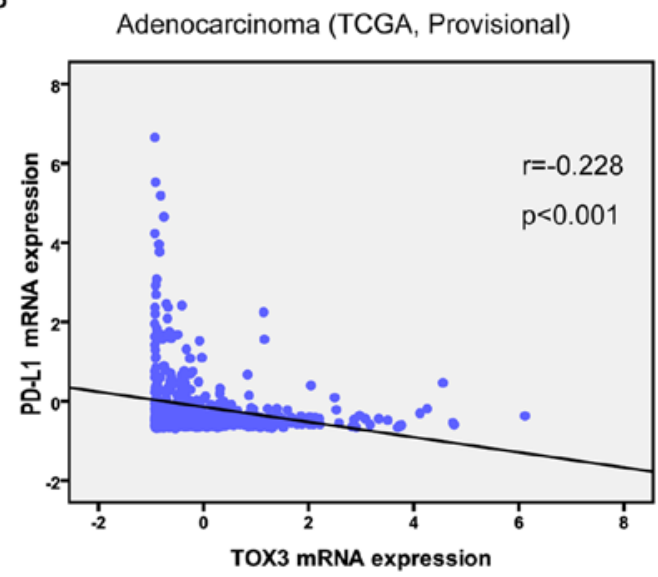

D

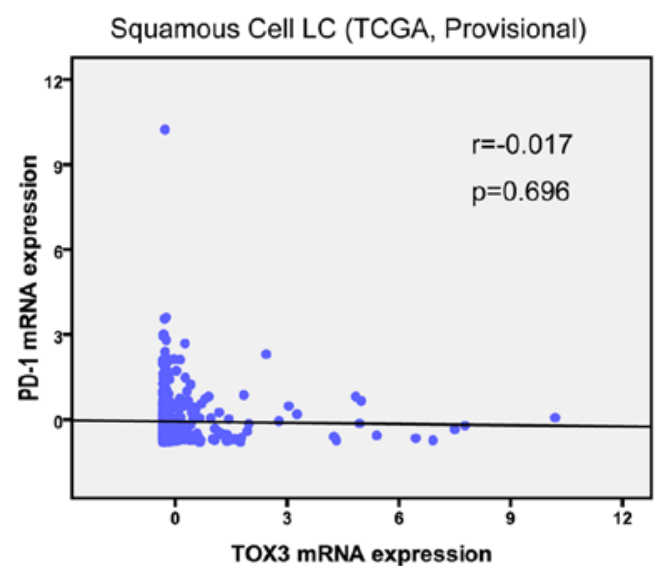

F

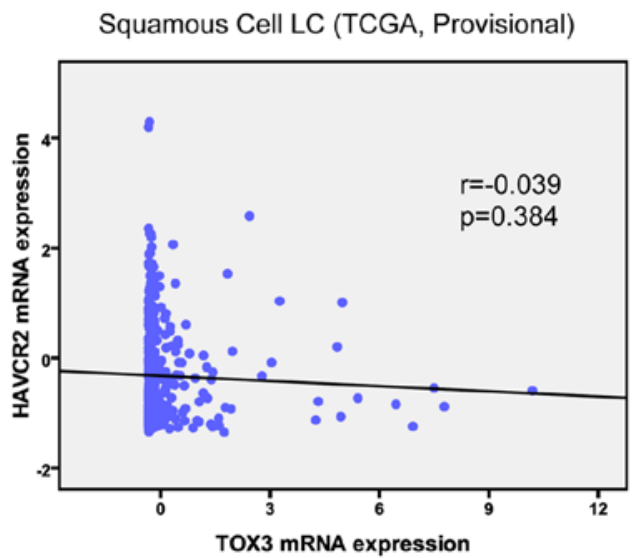

Figure 5. Increased expression of TOX3 is correlated with low expression of PD1, PD-L1 and HAVCR2 in lung adenocarcinoma. In the TCGA database, the Pearson's test was performed to analyze the correlation between TOX3 and a number of typical immunotherapy target genes. Gene correlation analysis in lung adenocarcinoma between TOX3 and (A) PD1, (B) PD-L1 and (C) HAVCR2. Gene correlation analysis between in squamous cell lung cancer between TOX3 and (D) PD1, (E) PD-L1 and (F) HAVCR2. TOX, thymocyte selection-associated high mobility group box; TCGA, The Cancer Genome Atlas; PD-1, programmed death-1 receptor; PD-L1, PD-ligand 1; HAVCR2, Hepatitis A virus cellular receptor 2.

performed in a previous study, it was indicated that TOX1 was silenced through $\mathrm{CpG}$ hypermethylation in lung cancer, which provides a possible mechanism for the development of lung cancer (13). The TOX3 gene is reported to be frequently absent in breast cancer, and has recently been demonstrated to be a risk factor for breast cancer (29). However, the role of TOX3 in lung cancer remains to be elucidated. In the present study, the analysis of several individual datasets derived from
Oncomine revealed that TOX3 was exclusively overexpressed in lung adenocarcinoma.

Further survival analysis with Kaplan-Meier plotter revealed that high levels of TOX3 mRNA were significantly associated with increased FP and OS in patients with lung adenocarcinoma. Based on these results, it was extrapolated that TOX3 may serve a tumor-suppressive role in lung cancer. This hypothesis was supported by Riaz et al (30), who 
indicated that TOX3 may act as a tumor-suppressor gene, as the risk allele rs3803662 was significantly associated with low TOX3 expression in breast cancer. Additionally, TOX3 knockdown increased cell proliferation in breast cancer. In addition, the TOX3 rs3803662 CT/TT genotype was associated with improved survival in patients with diffuse-type gastric cancer, acting as an independent prognostic marker (14). Notably, to the best of our knowledge, the present study is the first to report that high TOX3 transcription levels predict improved survival in patients with lung cancer who have received chemotherapy. It was hypothesized that TOX3 may be an important indicator of chemosensitivity in lung cancer.

In the past few years, emerging evidence pertaining to the association between the immune system and cancer has contributed to our understanding of the human immune system, which can eradicate cancer cells when properly stimulated (31). To date, the best characterized and most clinically studied immune checkpoints are PD-1, PD-L1 and CTLA-4 $(32,33)$. The PD-1 receptor is expressed on the surface of cells involved in the antitumor immune response (34). When its high-affinity ligand $\mathrm{PD}-\mathrm{L} 1$ binds to the $\mathrm{PD}-1$ receptor, the receptor complex activation induces intracellular inhibitory signaling cascades that result in dysfunctional tumor-infiltrating $\mathrm{T}$ cells (28). These $\mathrm{T}$ cells have exhibited poor proliferation and a reduced capacity to produce cytokines (32). PD-L1 tumor expression and pre-existing tumor T-cell infiltration have been correlated with improved clinical outcomes to anti-PD-1/anti-PD-L1 (35). Identifying genes or co-factors associated with these immunotherapy targets could increase our knowledge of immunotherapy and aid the identification of novel immunotherapeutic targets.

Correlation analysis using the cBioPortal database revealed that TOX3 is negatively correlated with PD-1, PD-L1 and HAVCR2 in lung adenocarcinoma. However, no significant correlation was observed between TOX3 and PD-1, PD-L1 or HAVCR2 in the squamous cell carcinoma subtype. PD-L1 expression has been recognized as the most important biomarker for predicting the treatment response of immunotherapy in lung cancer (4). The strong correlation between TOX3 and PD-L1 expression was in line with the prognostic value of TOX3 in lung adenocarcinoma, and indicated that TOX3 may serve as an immunomodulatory co-factor with a suppressive role in the development of lung adenocarcinoma.

HAVCR2, also known as Tim-3, is a newly identified immunity regulator that was reported to be ubiquitously expressed in multiple tumor cells and T cells (36). Similar to PD-1, HAVCR2 is also one member of inhibitory checkpoint molecules (37). HAVCR2 protein negatively regulates $\mathrm{Th} 1 / \mathrm{Tc} 1$ function by inducing cell death upon interaction with its ligand, galectin-9 (38). It has been demonstrated in previous studies that in vivo HAVCR2 blockade with other checkpoint inhibitors enhances antitumor immunity and suppresses tumor growth in a number of preclinical tumor models, which strongly indicate a potential role in tumor progression $(39,40)$. In the present correlation analysis, it was observed that TOX3 expression is negatively associated with HAVCR2 in lung adenocarcinoma. However, no significant correlation between TOX3 and HAVCR2 expression was detected in the squamous cell carcinoma subtype. This was consistent with the prognostic values of TOX3, which are conversely associated with HAVCR2 expression, indicating the tumor-suppressive role of TOX3 in lung adenocarcinoma.

In summary, ectopic TOX expression is common in lung cancer. In particular, elevated TOX3 expression defines a subgroup of patients with lung adenocarcinoma and predicts improved survival outcomes. Furthermore, TOX3 expression is negatively correlated with the immunotherapy targets PD-1, PD-L1 and HAVCR2 in lung adenocarcinoma, indicating that it serves a role in immunity activation. Analyses of TOX family members in a variety of databases, particularly those focused on their association with immunotherapeutic targets, offer a novel insight into the role of TOXs in lung cancer and provides a theoretical basis for future research in this field.

\section{Acknowledgements}

Not applicable.

\section{Funding}

The Natural Science Foundation of Guangdong Province, China (grant no. 2018A030313562), the Youth Innovative Talent Project of Colleges and Universities in Guangdong Province, China (grant no. 2017KQNCX073).

\section{Availability of data and materials}

The datasets used and/or analyzed during the current study are available from the corresponding author on reasonable request.

\section{Authors' contributions}

DZ and HYL conceived and designed the study. JXC and WQL analyzed and interpreted the data. DZ and HYL wrote the manuscript and DZ approved the final version to be submitted.

\section{Ethics approval and consent to participate}

Not applicable.

\section{Patient consent for publication}

Not applicable.

\section{Competing interests}

The authors declare that they have no competing interests.

\section{References}

1. Mayekar MK and Bivona TG: Current landscape of targeted therapy in lung cancer. Clin Pharmacol Ther 102: 757-764, 2017.

2. Somasundaram A and Burns TF: The next generation of immunotherapy: Keeping lung cancer in check. J Hematol Oncol 10: 87, 2017.

3. Khanna P, Blais N, Gaudreau PO and Corrales-Rodriguez L: Immunotherapy comes of age in lung cancer. Clin Lung Cancer 18: 13-22, 2017.

4. Wehler T, Wehler B and Stehle I: Immunotherapy in lung cancer: Checkpoint inhibitors. Deutsche medizinische Wochenschrift 140: 1835-1838, 2015 (In German). 
5. Quaratino S, Forssmann U and Marschner JP: New approaches in immunotherapy for the treatment of lung cancer. Curr Top Microbiol Immunol 405: 1-31, 2017.

6. Ettinger DS: Immunotherapy for lung cancer: Many questions yet to be answered. J Natl Compr Canc Netw 14: 935-938, 2016.

7. Goubet AG, Livartowski A and Romano E: Immunotherapy in lung cancer: New concepts. Rev Mal Respir 35: 642-651, 2018 (In French).

8. Sánchez de Cos Escuín J: New immunotherapy and lung cancer. Arch Bronconeumol 53: 682-687, 2017 (In English, Spanish).

9. Seehus CR and Kaye J: The role of TOX in the development of innate lymphoid cells. Mediators Inflamm 2015: 243868, 2015.

10. Yu X and Li Z: TOX gene: A novel target for human cancer gene therapy. Am J Cancer Res 5: 3516-3524, 2015.

11. Han CC, Yue LL, Yang Y, Jian BY, Ma LW and Liu JC: TOX3 protein expression is correlated with pathological characteristics in breast cancer. Oncol Lett 11: 1762-1768, 2016.

12. Schrader AM, Jansen PM and Willemze R: TOX expression in cutaneous T-cell lymphomas: An adjunctive diagnostic marker that is not tumour specific and not restricted to the CD4(+) CD8(-) phenotype. Br J Dermatol 175: 382-386, 2016.

13. Tessema M, Yingling CM, Grimes MJ, Thomas CL, Liu Y, Leng S, Joste N and Belinsky SA: Differential epigenetic regulation of TOX subfamily high mobility group box genes in lung and breast cancers. PLoS One 7: e34850, 2012

14. Zhang X, Zhu H, Wu X, Wang M, Gu D, Gong W, Xu Z, Tan Y, Gong Y, Zhou J, et al: A genetic polymorphism in TOX3 is associated with survival of gastric cancer in a Chinese population. PLoS One 8: e72186, 2013.

15. Wu W, Wagner EK, Hao Y, Rao X, Dai H, Han J, Chen J, Storniolo AMV, Liu Y and He C: Tissue-specific co-expression of long non-coding and coding RNAs associated with breast cancer. Sci Rep 6: 32731, 2016.

16. Rhodes DR, Yu J, Shanker K, Deshpande N, Varambally R, Ghosh D, Barrette T, Pandey A and Chinnaiyan AM: ONCOMINE: A cancer microarray database and integrated data-mining platform. Neoplasia 6: 1-6, 2004.

17. Rhodes DR, Kalyana-Sundaram S, Mahavisno V, Varambally R, Yu J, Briggs BB, Barrette TR, Anstet MJ, Kincead-Beal C, Kulkarni P, et al: Oncomine 3.0: Genes, pathways, and networks in a collection of 18,000 cancer gene expression profiles. Neoplasia 9: 166-180, 2007.

18. Lin HY, Zeng, Liang YK, Wei XL and Chen CF: GATA3 and TRPS1 are distinct biomarkers and prognostic factors in breast cancer: Database mining for GATA family members in malignancies. Oncotarget 8: 34750-34761, 2017.

19. Györffy B, Lanczky A, Eklund AC, Denkert C, Budczies J, Li Q and Szallasi Z: An online survival analysis tool to rapidly assess the effect of 22,277 genes on breast cancer prognosis using microarray data of 1,809 patients. Breast Cancer Res Treat 123: 725-731, 2010

20. Gao J, Aksoy BA, Dogrusoz U, Dresdner G, Gross B, Sumer SO, Sun Y, Jacobsen A, Sinha R, Larsson E, et al: Integrative analysis of complex cancer genomics and clinical profiles using the cBioPortal. Sci Signal 6: pl1, 2013.

21. Stearman RS, Dwyer-Nield L, Zerbe L, Blaine SA, Chan Z, Bunn PA Jr,Johnson GL, Hirsch FR, MerrickDT,Franklin WA, etal: Analysis of orthologous gene expression between human pulmonary adenocarcinoma and a carcinogen-induced murine model Am J Pathol 167: 1763-1775, 2005.

22. Landi MT, Dracheva T, Rotunno M, Figueroa JD, Liu H, Dasgupta A, Mann FE, Fukuoka J, Hames M, Bergen AW, et al: Gene expression signature of cigarette smoking and its role in lung adenocarcinoma development and survival. PLoS One 3: e1651, 2008.

23. Garber ME, Troyanskaya OG, Schluens K, Petersen S, Thaesler Z, Pacyna-Gengelbach M, van de Rijn M, Rosen GD, Perou CM, Whyte RI, et al: Diversity of gene expression in adenocarcinoma of the lung. Proc Natl Acad Sci USA 98: 13784-13789, 2001.
24. Hou J, Aerts J, den Hamer B, van Ijcken W, den Bakker M, Riegman P, van der Leest C, van der Spek P, Foekens JA, Hoogsteden HC, et al: Gene expression-based classification of non-small cell lung carcinomas and survival prediction. PLoS One 5: e10312, 2010.

25. Du L, Herbst RS and Morgensztern D: Immunotherapy in lung cancer. Hematol Oncol Clin North Am 31: 131-141, 2017.

26. Valecha GK, Vennepureddy A, Ibrahim U, Safa F, Samra B and Atallah JP: Anti-PD-1/PD-L1 antibodies in non-small cell lung cancer: The era of immunotherapy. Expert Rev Anticancer Ther 17: 47-59, 2017.

27. Seksenyan A, Kadavallore A, Walts AE, de la Torre B, Berel D, Strom SP, Aliahmad P, Funari VA and Kaye J: TOX3 is expressed in mammary $\mathrm{ER}(+)$ epithelial cells and regulates ER target genes in luminal breast cancer. BMC Cancer 15: 22, 2015.

28. Li L, Guo G, Wang F, Lv P, Zhu M, Gu Y, Han M and Pei X: TOX high mobility group box family member 3 rs3803662 and breast cancer risk: A meta-analysis. J Cancer Res Ther 14 (Suppl): S208-S212, 2018

29. Zhang Y, Cai P, Liang T, Wang L and Hu L: TIM-3 is a potential prognostic marker for patients with solid tumors: A systematic review and meta-analysis. Oncotarget 8: 31705-31713, 2017.

30. Riaz M, Berns EM, Sieuwerts AM, Ruigrok-Ritstier K, de Weerd V, Groenewoud A, Uitterlinden AG, Look MP, Klijn JG, Sleijfer S, et al: Correlation of breast cancer susceptibility loci with patient characteristics, metastasis-free survival, and mRNA expression of the nearest genes. Breast Cancer Res Treat 133: 843-851, 2012.

31. Nishioka Y: Cancer immunotherapy as a promising fourth standard therapy for lung cancer: Beyond 20 years for the development of immunotherapy. Respir Investig 54: 297, 2016.

32. Dal Bello MG, Alama A, Coco S, Vanni I and Grossi F: Understanding the checkpoint blockade in lung cancer immunotherapy. Drug Discov Today 22: 1266-1273, 2017.

33. Chae YK, Arya A, Iams W, Cruz M, Mohindra N, Villaflor V and Giles FJ: Immune checkpoint pathways in non-small cell lung cancer. Ann Transl Med 6: 88, 2018.

34. Berghmans T, Grigoriu B, Sculier JP and Meert AP: Immune checkpoint inhibitors (antibodies to PD1 and PD-L1), a new therapeutic weapon against non-small cell bronchial carcinoma. Rev Mal Respir 35: 197-205, 2018 (In French).

35. Berghmans T and Meert AP: Immunotherapy and non-small cell lung cancer: A (r)evolution. Rev Med Brux 38: 175-177, 2017 (In French)

36. Anderson AC: Tim-3: An emerging target in the cancer immunotherapy landscape. Cancer Immunol Res 2: 393-398, 2014.

37. Liu F, Zeng G, Zhou S, He X, Sun N, Zhu X and Hu A: Blocking Tim-3 or/and PD-1 reverses dysfunction of tumor-infiltrating lymphocytes in HBV-related hepatocellular carcinoma. Bull Cancer 105: 493-501, 2018.

38. Gao J, Qiu X, Li X, Fan H, Zhang F, Lv T and Song Y: Expression profiles and clinical value of plasma exosomal Tim-3 and Galectin-9 in non-small cell lung cancer. Biochem Biophys Res Commun 498: 409-415, 2018.

39. Das M, Zhu C and Kuchroo VK: Tim-3 and its role in regulating anti-tumor immunity. Immunol Rev 276: 97-111, 2017.

40. Yu M, Lu B, Liu Y, Me Y, Wang L and Zhang P: Tim-3 is upregulated in human colorectal carcinoma and associated with tumor progression. Mol Med Rep 15: 689-695, 2017.

This work is licensed under a Creative Commons Attribution-NonCommercial-NoDerivatives 4.0 International (CC BY-NC-ND 4.0) License. 